\title{
Socially-induced infertility in Ansell's mole-rats: are there depressed hormone levels in non-reproductive males and females?
}

\author{
P.R. de Bruin ${ }^{1}$, H. Viljoen ${ }^{1}$, A.M. Sichilima ${ }^{1}$ and N.C. Bennett ${ }^{2}$ \\ ${ }^{1}$ Department of Zoology and Entomology, University of Pretoria, Pretoria 0002 \\ ${ }^{2}$ Mammal Research Institute, Department of Zoology and Entomology, University of \\ Pretoria, Pretoria 0002
}

Ansell's mole-rat, Fukomys anselli, is a social subterranean mammal and exhibits an extreme reproductive division of labour. Reproduction in the colony appears to be restricted usually to a single female. Complete colonies captured throughout an entire calendar year were euthanased and the histology of the gonads and plasma hormone concentrations were measured in reproductive and non-reproductive members of both sexes. In males, the circulating levels of testosterone were highest in the reproductive male. The mean testes mass for male corrected for body mass was lower than that of the non-reproductive male. However, the mean testes volume of reproductive males was greater than that for the non-reproductive males and the seminiferous tubule diameter was greater in reproductive males. Reproductive females characteristically possessed corpora lutea of ovulation and pregnancy in their ovaries and this was met with much elevated progesterone concentrations in these females. In contrast, non-reproductive females showed a complete range of follicular genesis, but they did not possess corpora lutea of ovulation or pregnancy, in turn they show depressed progesterone concentrations. The current available evidence suggests that in Ansell's mole-rats, the non-reproductive males and females refrain from sexual activity by being subordinate and moreover related to the breeding pair.

Keywords: Reproduction, progesterone, testosterone, testes, ovaries, 


\section{Introduction}

The family Bathyergidae is characterised by a broad spectrum of social organisation which has resulted from ecological constraints shaping social evolution in those species that occupy arid and harsh environments. At the one end of the spectrum is the solitary mole-rats (the genera, Bathyergus, Georychus and Heliophobius) whereas other species exhibit varying degrees of colonial and social development (the genera Cryptomys, Fukomys and Heterocephalus) (Bennett \& Faulkes, 2000) Socially is characterised by the development of a reproductive division of labour in which a small subset of each sex are responsible for procreation. The remaining non-reproductive animals in the colony are reproductively quiescent but not sterile, performing work related tasks that maintain the integrity of the colony (Bennett, Faulkes \& Jarvis, 1999). It has been postulated that socially has arisen is some mole-rat species as a result of two extrinsic factors acting upon the mole-rats. The Aridity Food Distribution Hypothesis posits that species which occur in arid environments in which the food resource is unequally distributed must be social in order to extend their burrow systems extensively during periods of rainfall, so called windows of opportunity (Jarvis, O'Riain, Bennett \& Sherman, 1994). In contrast, the solitary species which occupy generally more mesic environments have longer periods during which they may forage efficiently because of equally distributed food resources and higher rainfall (Jarvis et al. 1994, Bennett et al. 1999).

In the social species of mole-rat, reproduction is unequally partitioned and the colonies exhibit reproductive skew (sensu Vehrencamp, 1983). Reproductive skew is defined as the unequal distribution of direct reproduction among same-sex members of a social group (Vehrencamp, 1983; Keller and Reeve 1994). Social societies may demonstrate many forms of reproduction ranging from egalitarian, where all individuals breed, to despotic, where only one dominant animal breeds and the others are reproductively suppressed (Keller and Reeve 1994). African mole-rats exhibit a broad range of cooperative breeding mechanisms and overall skew in females is much higher than that in males, since several putative males may be responsible for procreation (Bishop et al, 2002; Burland et al 2004. Again interspecies differences occur in female reproductive skew, where some species may occasionally exhibit plural breeding and others show strictly omnipotent rule by a single breeding queen (Bennett \& Jarvis, 1988; Bennett et al., 1997, Bennett et al. 1999; Faulkes \& Bennett, 2007, Sichilima et al. 2008). 
Snowdon (1996) proposed two opposing models to explain reproductive suppression in cooperatively breeding animals. The first is the dominant control model (DCM) which posits that a dominant breeding animal forcibly down-regulates the hypothalamo-pituitary-gonadal axis of the subordinates through aggressive interactions with subordinates. The breeder gains from recruiting helpers to assist in the raising of offspring and the gathering of food (Snowden 1996). Here, both behavioural and olfactory signals play a role in the control process. Experiments undertaken on naked mole-rats by Reeve (1992) and Faulkes et al. (1990) suggest that reproductive control is through dominant control by the queen as a result of aggressive interactions in the form of pushing and shoving that is directed towards the non-reproductive colony members. The second model is the self-restraint model (SRM), which posits that subordinates exercise physiological restraint from within given the possibility of threat by a dominant and simply refrain from breeding attempts. Other factors that may also play a role in the SRM are resource availability, the number of helpers as well as breeding experience (Snowden 1996). Clarke et al. (2001) have intimated that in the non reproductive male and female Damaraland mole-rat, Fukomys damarensis the SRM is operational. Many other social species of mole-rat belonging to the genus Cryptomys do not possess specific physiological blocks to reproduction and skew in this instance is maintained solely by incest avoidance (Bennett et al. 1997)

The social genera of African mole-rats, encompass a number of species that range in both the degree of sociality exhibited and habitat type encountered (Bennett et al. 1999). The two most social species, the naked mole-rat, Heterocephalus glaber, and the Damaraland mole-rat, $F$. damarensis, possess relatively large colonies with a distinct reproductive division of labour and a marked work related division of labour into frequent workers and non-workers (Bennett and Jarvis 1988, Jarvis 1991), they also occur in very arid habitats. Within the genera Fukomys and Cryptomys are a number of social cooperatively breeding species with a distinct reproductive division of labour, but not such a clearly demarcated work division of labour (Wallace and Bennett 1998, Moolman et al. 1998) and they tend to inhabit more mesic environments, where soil is workable for longer periods of time during the year.

Individuals within a social group are usually closely related, comprising of the breeding animals and their offspring and in such instances, the avoidance of inbreeding is important 
(Bennett et al. 1997; Greeff and Bennett 2000). Oosthuizen et al. (2008) found that in the Natal mole-rat, Cryptomys hottentotus natalensis, inbreeding avoidance is the main force driving reproductive repression in the subordinates. Physiological evidence from circulating basal LH concentrations and the response of the pituitary to an exogenous GnRH challenge revealed that in colonies of the Mashona mole-rat, Fukomys darlingi, a close relative of Ansell's mole-rat, repression is through incest avoidance (Bennett et al. 1997).

Behavioural contact in the form of shoving and pushing events by the queen of a colony has been found to be very important in the suppression of reproduction in the non-breeding males and females of naked mole-rats (Faulkes 1990). Indeed, there is little evidence of chemical control through the use of primer pheromones (Faulkes and Abbott 1993). So marked is the physiological suppression in the naked mole-rat that follicular development is inhibited and does not extend beyond the pre-antral stage with the result of the subordinate females' failing to ovulate (Faulkes et al. 1990). Indeed, suppression of reproduction is present in both nonbreeding male and female naked mole-rats (Faulkes and Abbott 1991). Furthermore, although spermatogenesis occurs in the non-breeding males, the plasma concentration of luteinizing hormone (LH) and urinary testosterone levels are lower than that of the breeding males. When the subordinate males are removed from the colony and housed separately or with an unrelated female, their urinary testosterone and plasma LH levels increased (Faulkes and Abbott 1991).

Recent work on the giant mole-rat, Fukomys mechowii, a close cousin of the Ansell's mole-rat on the phylogenetic tree, has shown this species to exhibit similar colony sizes as those reported for Ansell's mole-rat 9 (range 6-16) (Sichilima 2008). The non-reproductive animals of both sexes are not physiologically suppressed (Bennett, Molteno \& Spinks, 2000). Reproductive relaxation within colonies appears in the wet season, November to April, where plural breeding females are found in colonies, but at no other time of the year does this phenomenon occur (Sichilima, Faulkes \& Bennett, 2008).

Ansell's mole-rat, Fukomys anselli is endemic to the Miombo woodlands of the Zambian region, mainly found around the suburbs of Lusaka and the adjacent region but not past the Kafue River (H. Burda and N.C. Bennett, unpublished data). Ansell's mole-rats are social, occurring in family groups of about 9 individuals (range 6-15), which comprise of the breeding male and female with several of their offspring (Burda 1989, Sichilima et al. submitted). 
Laboratory studies have found that offspring can be produced throughout the year, consisting of two pups (range from 1-5), intimating that breeding is aseasonal (Burda 1989, 1990, Begall and Burda 1998).

Willingstorfer et al. (1998) investigated ovarian activity in female Ansell's mole-rats of varying age and reproductive status, interestingly they found that non-breeding females show follicular genesis up until the tertiary follicle stage. In contrast, reproductive females possessed both Graafian follicles and corpora lutea of pregnancy. This study was from laboratory reared animals and did not investigate the male members of the colony.

We used Ansell's mole-rats to address several questions related to their reproduction and physiology. The aims of the study were to a) determine if the reproducing female from respective colonies captured throughout April 2009 to August 2009 possess corpora lutea of ovulation and pregnancy in their ovaries. b) we wanted to elucidate if the non-reproducing females exhibit ovulatory activity, by the presence of corpora lutea of ovulation in the ovary, whilst in the confines of the colony, c) to ascertain whether the reproducing female has higher circulating plasma progesterone levels when compared to the non-reproductive females. In the male individuals we want to investigate if $d$ ) the testicular mass and volume were greater in the reproductive males e) the seminiferous tubule diameter of the testes is larger in reproductive males and finally e) investigate whether the circulating plasma testosterone levels of the reproducing males are higher than those of the non-reproducing males.

We hypothesised that since Ansell's mole-rats occur in a predominantly mesic environment that non-reproductive females whilst in the confines of the colony will refrain from mating within the colony due to incest avoidance mechanisms and that the females would show depressed concentrations of circulating progesterone due to them being anovulatory. We further hypothesized that non-reproductive males would refrain from sexual activity while in the confines of the colony and would potentially exhibit reduced testosterone titres in comparison to their reproductive male counterparts.

\section{Materials and Methods}

Subjects and sample collection

The burrow systems of Ansell's mole-rats were located and the tunnels excavated using modified Hickman live traps baited with sweet potato to capture the mole-rats (Hickman 1979). The traps 
were set at the openings of the burrow system and covered with a soil layer to prevent any light from entering the tunnels and subsequently to keep the traps cool. The traps were checked every two to four hours so that the animals could be removed as soon as possible. All the members of an entire colony were captured before the traps were finally removed. The individual colony members were exposed to an overdose of chloroform until expiration. Reproductive males were identified by staining around the mouth and bulging abdominal testes. Reproductive females were clearly identified by a perforate vagina and elongated teats in the axillary and inguinal regions. The gonads of the male and female animals were then removed and placed in buffered $10 \%$ formalin for four days. The gonads were later removed and permanently preserved in $70 \%$ ethanol.

\section{Histological procedure}

The mass of the ovaries and testes were determined to the nearest $1 \mathrm{mg}$ using a Sartorius 1213MP scale (Sartorius AG, Goettengen, Germany). The mass of the paired ovaries and testes were averaged. The length of the ovaries and testes were recorded to the nearest $0.01 \mathrm{~mm}$ using a Vernier caliper (Mitutoyo American Corporation, Aurora, Illinois). The tissue samples were dehydrated by exposing each sample to a series of alcohol baths. The samples were then embedded in paraffin wax (Drury and Wallington 1967) and each ovary was cut into $7 \mu \mathrm{m}$ sections using a microtome. The samples were mounted on untreated microscope slides with albumin. The same procedure was followed for the testes, however only 20-30 sections from the centre of the testes were used. Haemotoxylin was used to stain all the sections with eosin as the counter stain.

Ovarian histology

Bennett et al. (1994) was used to classify each follicle. The follicles were identified, classified and counted. The ovarian volume was calculated to the nearest $0.1 \mathrm{~mm}^{3}$ by using the formula for an ellipsoid: $\mathrm{V}=4 / 3 \pi \mathrm{ab}^{2}$.

\section{Testicular histology}

Sections of $7 \mu \mathrm{m}$ were made for the testes and examined under a 20x magnification. An ocular micrometer was used to measure randomly selected, cross-sectioned seminiferous tubules. The 
diameters of the seminiferous tubules were measured from ten randomly selected tubules in each testis of every male. The testicular volume was determined by using the formula for an ellipsoid (see above).

\section{Testosterone assay}

The plasma testosterone $(\mathrm{T})$ concentrations of both reproductive and non-reproductive males were determined to elucidate if the non-reproductive males had reduced levels of T. A Coat-aCount total testosterone kit (Diagnostic Products Corporation, Los Angeles, California) was used to determine the plasma testosterone levels. The antiserum is highly specific for testosterone and has a low cross-reactivity with other naturally occurring steroids, except dihydrotestosterone, which is $<5 \%$. The assay was validated for Ansell's mole-rats by comparing the slope of the curve produced with a double dilution from unextracted mole-rat plasma (over the range 1:1 to 1:64) against a standard curve of known calibrators, parallelism was exhibited.

\section{Progesterone assay}

The plasma progesterone $(\mathrm{P})$ concentrations of the reproductive and non-reproductive females were measured to ascertain if the non-reproductive females have reduced levels of $P$, this in association with an absence or presence of corpora lutea enabled us to determine if ovulation has taken place or not. The $\mathrm{P}$ concentrations were determined using Coat-a-Count progesterone kits (Diagnostic Products Corporation, Los Angeles, California). The antiserum is highly specific for progesterone with a low cross-reactivity to all other naturally occurring steroids except 20 adihydroprogesterone and 11-deoxycortisol with a cross reactivity of $2 \%$ and $2.4 \%$ respectively. The assay was validated for Ansell's mole-rats by comparing the slope of the curve produced with a double dilution from unextracted mole-rat plasma (over the range 1:1 to 1:64) against a standard curve of known calibrators, parallelism was exhibited.

Statistical analysis

Statistica 9.0 (Stat Soft, Inc., Tulsa, Oklahoma) was used to do the statistical analysis. We tested for normality after which an independent $t$-test with variables was done in order to determine if there was a significant difference between the body mass, gonadal volume, seminiferous tubule diameter and testosterone concentration of reproductive and non-reproductive males as well as 
body mass, gonadal volume, number of follicles and progesterone concentration of reproductive and non-reproductive females. The results are presented as means $\pm \mathrm{SE}$.

\section{Results}

The mean body mass of the reproductive males $(81.45 \pm 13.71 \mathrm{~g}, \mathrm{n}=18)$ was significantly greater than the mean body mass of the non-reproductive males $(39.80 \pm 18.97 \mathrm{~g}, \mathrm{n}=49)(t$-test $=$ $8.51, \mathrm{p}<0.01)$. The reproductive females also had a significantly greater mean body mass $(63.87$ $\pm 11.39 \mathrm{~g}, \mathrm{n}=19)$ than that of the non-reproductive females $(33.47 \pm 11.78 \mathrm{~g}, \mathrm{n}=64)(t$-test $=8.25, \mathrm{p}$ $<0.01)$.

After we corrected for body mass we found that there was no significant difference between the mass of the testes from the reproductive males $(0.580 .62 \mathrm{mg} / \mathrm{g}, \mathrm{n}=12)$ compared with the testes of the non-reproductive males $\left(0.79 \_0.61 \mathrm{mg} / \mathrm{g}, \mathrm{n}=37\right)$. ( $\mathrm{t}$-test $\left.=0.25, \mathrm{P}=0.80\right)$. The same was found when we compared the ovarian mass of the reproductive females $\left(\begin{array}{ll}0.22 & 0.29\end{array}\right.$ $\mathrm{mg} / \mathrm{g}, \mathrm{n}=13)$ to that of the non-reproductive females $(0.200 .25 \mathrm{mg} / \mathrm{g}, \mathrm{n}=42)(\mathrm{t}-\mathrm{test}=0.21$, $\mathrm{P}=0.84)$.

There was no significant difference between the mean gonadal volume of the reproductive males $\left(0.08 \pm 0.05 \mathrm{~mm}^{3}, \mathrm{n}=11\right)$ when compared to the mean gonadal volume of the non-reproductive males $\left(0.04 \pm 0.03 \mathrm{~mm}^{3}, \mathrm{n}=38\right)(t$-test $=1.74, \mathrm{p}=0.1)$. The same pattern was seen when we compared the mean gonadal volume for the reproductive females $(0.02 \pm$ $\left.0.03 \mathrm{~mm}^{3}, \mathrm{n}=12\right)$ with the mean gonadal volume of the non-reproductive females $(0.01 \pm$ $\left.0.01 \mathrm{~mm}^{3}, \mathrm{n}=45\right)(t$-test $=1.53, \mathrm{p}=0.14)$.

The mean diameter of the seminiferous tubules of the reproductive males $(154.91 \pm$ $40.38 \mu \mathrm{m}, \mathrm{n}=11$ ) was significantly greater than the diameter of the non-reproductive males $(114.80 \pm 38.92 \mu \mathrm{m}, \mathrm{n}=38)(t$-test $=11.87, \mathrm{p}<0.01)$.

The testosterone of the reproductive males had a significantly higher concentration (7.09 $\left.\pm 3.81 \mathrm{mmol}^{-1} \mathrm{~L}^{-1} \mathrm{n}=17\right)$ than that of the non-reproductive males $\left(1.00 \pm 0.87 \mathrm{mmol} . \mathrm{L}^{-1}, \mathrm{n}=47\right)(t$ test $=6.76, \mathrm{p}<0.01)$.

There was no significant difference between the number of primary follicles found in the ovaries of reproducing females $(4.80 \pm 2.77, \mathrm{n}=12)$ and the non-reproducing females $(9.16 \pm$ 
$5.58, \mathrm{n}=45)(t$-test $=-1.67, \mathrm{p}=0.11)$, the same was true for the secondary/tertiary follicles of the reproductive $(8.20 \pm 1.92, \mathrm{n}=12)$ and non-reproductive females $(8.00 \pm 4.70, \mathrm{n}=45)(t$-test $=0.09$, $\mathrm{p}=0.93$ ). A significant difference was, however, found between the number of Graafian follicles found in the ovaries of the reproductive females $(2.60 \pm 2.97, n=12)$ and the non-reproductive females $(0.74 \pm 0.93, \mathrm{n}=45)(t$-test $=2.44, \mathrm{p}=0.02)$. There was also a significant difference in the number of corpora lutea present in the ovaries of reproductive females $(2.60 \pm 1.52, \mathrm{n}=12)$ and that of the non-reproductive females, who exhibited no corpora lutea of ovulation $(0.00 \pm$ $0.00, \mathrm{n}=45)(t$-test $=-8.00, \mathrm{p}<0.01)($ Figure 1$)$.

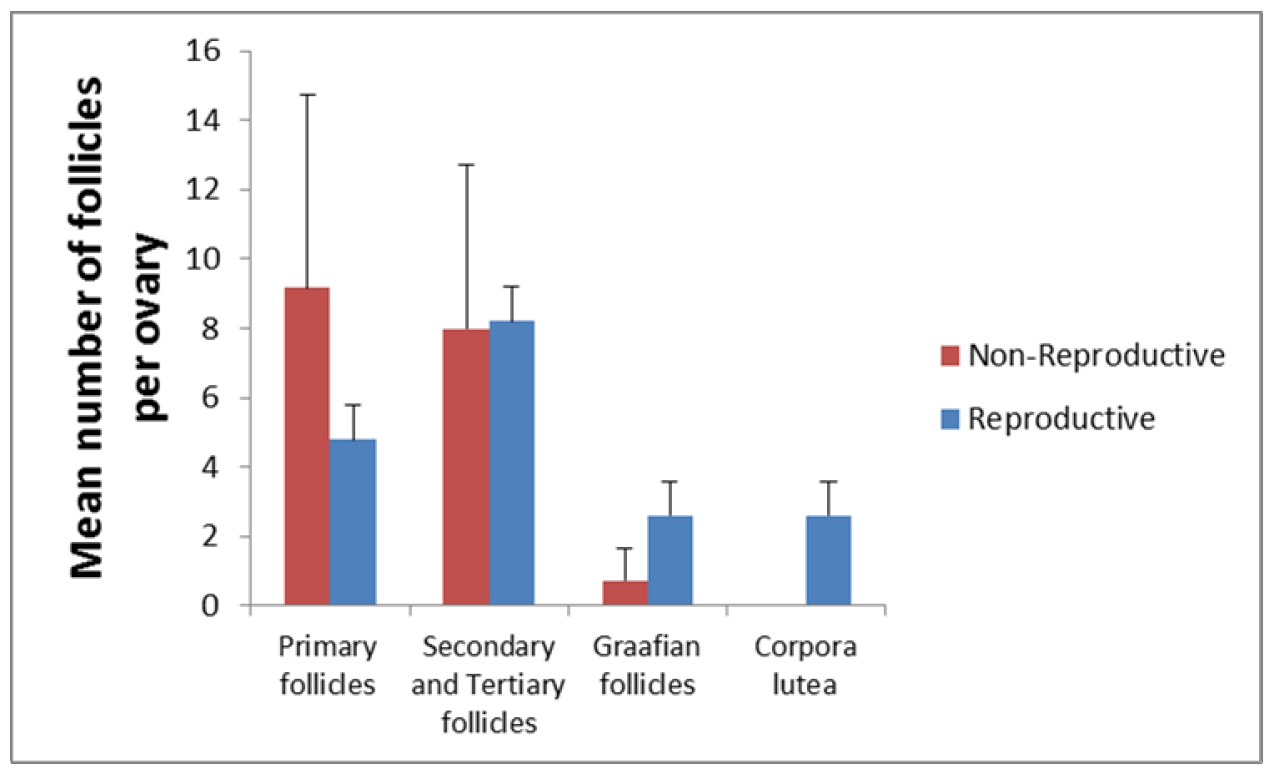

Figure 1. The mean \pm SD number of follicles per ovary of reproductive and non-reproductive female Ansell's mole-rats.

The progesterone concentration in the reproductive females $\left(34.00 \pm 40.32 \mathrm{mmol} . \mathrm{L}^{-1}\right.$, $\mathrm{n}=17)$ was significantly higher than in the non-reproductive females $\left(4.07 \pm 5.84 \mathrm{mmol}^{-1}\right.$, $\mathrm{n}=80)(t$-test $=5.04, \mathrm{p}<0.01)$.

\section{Discussion}

Colonies of Ansell's mole-rats were trapped out on a monthly basis and where possible the gonads and plasma were obtained from entire colonies. In all social Cryptomys and Fukomys species studied to date there is a marked reproductive skew characteristic of cooperatively breeding societies (Bennett et al. 1999). Usually a single reproductive female and 1 to 3 putative 
male consorts are responsible for procreation (Bennett and Jarvis 1988, Burda 1989, Bennett et al. 1994, Bennett and Aguilar 1995). In this study we were able to consistently demonstrate from the histology of the gonads and the circulating steroidal hormones of individuals from sacrificed colonies that a single reproductive female is responsible for procreation in the colony, whereas all other colony members appear to be non-reproductive, however, unusually it was found that during the wet season three cases of plural breeding were reported with a queen lactating and another pregnant. Plural breeding has been previously reported in the naked mole-rat and more rarely in the common mole-rat (Faulkes \& Bennett, 2007). Interestingly, from another study involving the capture of mole-rats over an entire calendar year, the giant mole-rat which also occurs in Zambia was also found to exhibit plural breeding with two reproductive females being present in the colony on several occasions during the wet season (Sichilima et al 2008b). This finding is significant since Sichilima et al. (submitted) found plural breeding in three colonies of Ansell's mole-rat at the end of the wet season. It would appear that during the rainy season there is a relaxation of reproductive skew in these central African mole-rats. Overall, in Ansell's molerat the reproductive male and female are usually the largest individuals of the respective sex. Indeed, the reproductive male is readily identified by being the largest animal in the colony.

The socially-induced infertility continuum posits that amongst outbred social mole-rats that inhabit mesic habitats, where opportunities for dispersal and of becoming a reproductive are high, the staying incentives offered by the breeders to non-breeders should be much higher than in those regions with strong environmental constraints (Bennett et al. 1997; Faulkes \& Bennett, 2007). As such, one would predict that reproductive inhibition would be solely through an incest avoidance component to social repression for mesic dwelling mole-rats. In marked contrast to the mesic species, the Damaraland mole-rat inhabits areas where the ecological constraints to successful dispersal are high and, as such, reproductive animals need to offer only few staying incentives to the non-reproductive colony members. Indeed, within this species the evolution of a physiological suppression appears operational in the non-reproductive female mole-rats. Behavioural repression is operational in non-reproductive Damaraland males but a strong physiological suppression is present in non-reproductive Damaraland females (Bennett 1994, Bennett et al. 1996). 
Since in Ansell's mole-rat colonies size are small, despite the fact that breeding occurs throughout the year, we propose that in colonies of Ansell's mole-rats reproductive repression is probably purely through incest avoidance as has been reported in a closely related species the Mashona mole-rat, Fukomys darling (Bennett et al. 1997). In the eusocial Damaraland mole-rats the testosterone profiles of reproductive males are indistinguishable from those of nonreproductive males (Bennett 1994). But in Ansell's mole-rat the reproductive male can be distinguished from the non-reproductive male by his elevated testosterone profile (this study).

The mean testes mass corrected for body mass is unexpected, but this can be explained as follows. If one examines the mean $\mathrm{n}=$ body mass of the reproductive animals compared to those of the non-reproductive animals it is observed that there is a cohort of non-reproductive animals missing around the 60-70g mass in males and 40-50g mass in females. We speculate that these animals are the dispersing animals that regularly leave the colony and pair up with unrelated conspecifics. The finding that the gonads of the smaller animals are per gram body mass larger than the breeders intimates that in these animals testicular growth occurs rapidly in the anticipation

The mean testes volume is larger but not significantly so in the reproductive male and this is probably the result of a more active testes. The mean seminiferous tubule diameter of the reproductive male was statistically greater than that of the non-reproductive male. The histological preservation did not allow us to investigate spermatogenesis in reproductive and non-reproductive males.

In the females, it is only the reproductive females who possess corpora lutea of ovulation or pregnancy in the ovaries, without exception the non-reproductive females lack these structures. However, it appears that the non-reproductive females are capable of undergoing follicular genesis and producing Graafian follicles, but these structures atrese and this may be the result of a lack of opportunities for copulation within the colony to mate with non kin or unfamiliar males that are absent in the colony. Willingstorfer et al. (1998) did not find Graafian follicles present in the ovaries of non-reproductive females, but in our study we found that nonreproductive females possessed Graafian follicles, but did not exhibit corpora lutea of ovulation. The mean circulating progesterone concentration in reproductive females was significantly higher than those of the non-reproductive females and this mirrors the histological findings. 
Thus it would appear that colonies of Ansell's mole-rat comprise of a single large breeding pair and a few much smaller non-reproductive animals that are the offspring of this pair. We speculate that if non-reproductive animals of either sex are of a sufficient body mass and rainfall events present opportunities for dispersal, these larger non-reproductive animals may disperse and establish new colonies by pairing with an unrelated and non-kin individual (this is currently being investigated). Indeed, an examination of the body mass distribution within colonies revealed that large animals did indeed leave the colony (A.M. Sichilima unpublished data).

The findings demonstrate that Ansell's mole-rat breeds throughout the year, with young being either in utero or having just been born during most months of the year, intimating that reproductive animals in the colony are primed for sexual activity throughout the year, in reproductive males this is demonstrated by high testosterone concentrations and in the reproductive females by the presence of corpora lutea of ovulation and pregnancy and much elevated progesterone concentrations. Reproductive repression in the non-reproductive colony members is presumably through incest avoidance since females show a full range of follicular genesis, but they fail to ovulate.

Bennett et al. (2010) have demonstrated that non-reproductive female Ansell's mole-rats removed from the confines of the colony undergo spontaneous ovulation, but while present in the colony this is absent. It is possible that these females may show reproductive restraint while in the presence of the breeder. Interestingly, non-reproductive males have very low testosterone concentrations and may also avoid copulations with siblings or their mother whilst in the colony. In the Damaraland mole-rat which is also an aseasonal breeder whose reproductive females exhibit spontaneous ovulation, the reproductive female orchestrates physiological suppression on the subordinates. In Damaraland mole-rats progesterone levels are very low or non-detectable in non-reproductive females and corpora lutea are absent and the pituitary LH concentrations and the response of the pituitary to a GnRH challenge is depressed (Bennett et al. 1993, 1994). In the males there is no difference in testosterone concentrations between reproductive and nonreproductive males (Bennett 1994).

Willingstorfer et al. (1998) hypothesised that non-reproductive females failed to produce corpora lutea of ovulation because of insufficient copulatory activity. We agree that this is 
probably the main reason that non-reproductive females do not ovulate in the confines of the colony. However, once removed from the colony the females undergo spontaneous ovulation when they pair up with an unrelated male and spontaneous ovulation occurs once again (Bennett et al. 2010). A similar pattern of reproductive inhibition is seen in the giant mole-rat, where nonreproductive females are anovulatory whilst in the confines of the colony but start to spontaneously ovulate when removed from the colony and housed on their own (Faulkes Sichilima, van Sandwyk, Lutermann and Bennett. 2010). Thus in the presence of the reproductive pair ovulation is repressed. In sum, the current available evidence suggests that in Ansell's mole-rats, the non-reproductive males and females refrain from sexual activity by being subordinate and moreover related to the breeding pair. We speculate that when these nonreproductive animals attain a critical body mass and sufficient rainfall has occurred that these individuals will disperse and establish new colonies by pairing with unrelated conspecifics. Relatedness coefficients of colony members are being investigated by Faulkes et al. (in prep).

\section{Acknowledgements}

We thank Babsie Potgieter for supplying all the laboratory equipment as needed during the study period. The research was funded by the SARCHI Chair of mammal behavioural ecology and physiology from the Department of Science and Technology and the National Research Foundation, South Africa (to N.C Bennett). The ethics committee of the University of Pretoria authorized the research. We also acknowledge a bursary from the NRF (to P.R de Bruin).

\section{References}

Begall, S and Burda, H. 1998. Reproductive characteristics and growth rate in the eusocial Zambian common mole-rat (Cryptomys sp., Bathyergidae). Zeitschrift fur Saugeteirkunde. 63, 297-306

Bennett, N.C. 1994. Reproductive suppression in social Cryptomys damarensis colonies: a lifetime of socially-induced sterility in males and females. Journal of Zoology, London. 234, 25 39

Bennett, N.C. and Aguilar, G.H. 1995. The reproductive biology of the giant Zambian mole-rat, Cryptomys mechowi (Rodentia: Bathyergidae). South African Journal of Zoology.30, 1-4 
Bennett, N.C.,Faulkes, C.G. and Jarvis, J.U.M. 1999. Socially-induced infertility incest avoidance and the monopoly of reproduction in cooperatively breeding African mole-rats. Advances in the study of Behaviour.28, 75-113

Bennett, N.C., Faulkes, C.G. and Molteno, A.J. 1996. Reproductive suppression in subordinate, non-breeding female Damaraland mole-rats: two components to a lifetime of socially-induced infertility. Proceedings of the Royal Society of London B.263, 1599-1603

Bennett, N.C., Faulkes, C.G. and Spinks, A.C. 1997. LH responses to single doses of exogenous GnRH by social Mashona mole-rats: a continuum of socially induced infertility, family Bathyergidae. Proceedings of the Royal Society of London B. 264, 1001-1006

Bennett, N.C. and Jarvis, J.U.M. 1988. The social structure and reproductive biology of colonies of the mole-rat Cryptomys damarensis (Rodentia, Bathyergidae). Journal of Mammalogy. 69, 293-302

Bennett, N.C., Jarvis, J.U.M., Faulkes, C.G. and Millar, R.P. 1993. L.H. responses to single doses of exogenous GnRH by freshly captured Damaraland mole-rats, Cryptomys damarensis. Journal of Reproduction and Fertility. 99, 81-86

Bennett, N.C., Jarvis, J.U.M., Millar, R.P., Sasano, H. and Ntshinga, K.V. 1994. Reproductive suppression in eusocial Cryptomys damarensis colonies: socially-induced infertility in females. Journal of Zoology, London.233, 617-630 .

Bennett, N.C., Molteno , A.J. \& Spinks, A.C. (2000). Pituitary sensitivity to exogenous GnRH in giant Zambian mole-rats, Cryptomys mechowi (Rodentia: Bathyergidae): support for the "socially-induced infertility continuum". Journal of Zoology, London 252: 447-452.

Bennett, N.C., van Sandwyk, J. and Lutermann, H. 2010. The pattern of ovulation in Ansell's mole-rats: phylogenetic or ecological constraints? Journal of Zoology.281, 66-73

Bishop, J.M., , O’Ryan, C., Spinks, A.C.,Bennett, N.C.. \& Jarvis, J.U.M. (2004). Molecular insight into patterns of colony composition and paternity in the common mole-rat, Cryptomys hottentotus hottentotus: a subterranean social mammal. Molecular Ecology 13: 1217-1229

Burda, H. 1989. Reproductive biology (behavioural, breeding and postnatal development) in subterranean Zambian mole-rats, Cryptomys hottentotus(Bathyergidae). Zeitschrift fur Saugeteirkunde. 54, 360-376

Burda, H. 1990. Constraints of pregnancy and evolution of sociality in mole-rats. With special reference to reproductive and social patterns in Cryptomys hottentotus (Bathyergidae, Rodentia). Zeitschrift fur zoologische Systematik und Evolutionsforschung.28, 26-39 
Burland, T.M., Bennett, N.C.., Jarvis, J.U.M. \& Faulkes, C.G. (2004). Colony structure and parentage in wild colonies of cooperatively breeding Damaraland mole-rats suggest incest avoidance alone may not maintain reproductive skew. Molecular Ecology 13: 2371-2379

Clarke, F.M., Miethe, G.H. and Bennett, N.C. 2001. Reproductive suppression in female damaraland mole-rats Cryptomys damarensis: dominant control or self-restraint? Proceedings of the Royal Society ofLondon B. 268, 899-909

Cooney, R. and Bennett, N.C. 2000. Inbreeding avoidance and the reproductive skew in a cooperative mammal. Proceedings of the Royal Society of London B. 267, 801-806

Drury, R.A.B. and Wallington, E.A. 1967. Carleton's histological technique. Oxford University Press, London, United Kingdom.

Faulkes, C.G. 1990. Social suppression of reproduction in the naked mole-rat, Heterocephalus glaber. PhD thesis. University of London.

Faulkes, C.G. and Abbott, D.H. 1991. Social control of reproduction in breeding and nonbreeding male naked mole-rats (Heterocephalus glaber). Journal for Reproduction and Fertility. 93, 427-435

Faulkes, C.G. \& Abbott, D.H. (1993). Evidence that primer pheromones do not cause social suppression of reproduction in male and female naked mole-rats (Heterocephalus glaber). Journal for Reproduction and Fertility.99, 225-230.

Faulkes, C.G., Abbott, D.H. and Jarvis, J.U.M. 1990. Social suppression of ovarian cyclicity in captive and wild colonies of naked mole-rats, Heterocephalu sglaber. Journal for Reproduction and Fertility.88, 559-568.

Faulkes, C.G. \& Bennett, N.C. (2007). Reproductive skew in African mole-rats: behavioral and physiological mechanisms to maintain high skew. Pp 369-396. In: Reproductive Skew in Vertebrates. Eds Hagar, R and Jones, C.B. Cambridge University Press, Cambridge. UK 523 p

Faulkes, C.G., Sichilima, . A.M. , Van Sandwyk, J, Lutermann, H. \& Bennett, N.C.. (2010). Control of ovulation in female giant mole-rats, Fukomys mechowii (Rodentia: Bathyergidae). Journal of Zoology, London. 282: 64-74

Greeff, J.M. and Bennett, N.C. 2000. Causes and consequences of incest avoidance in the cooperative breeding mole-rat, Cryptomys darlingi (Bathyergidae). Ecology Letters.3, 318-328

Hackländer, K., Mostle, E. and Arnold, W. 2003. Reproductive suppression in female Alpine marmots, Marmota marmot. Animal behaviour.65, 1133-1140

Hickman, G.C. 1979. A live-trap and trapping technique for fossorial mammals. South African Journal of Zoology.14, 9-12 
Jarvis, J.U.M. , O’Riain, M.J., Bennett, N.C. \& Sherman, P.W. (1994). Mammalian eusociality - a family affair. Trends in Ecology and Evolution 9: 47-51

Keller, L. and Reeve, H.K. 1994. Partitioning of reproduction in animal societies. Trends in Ecology and Evolution.9, 98-102

Moolman, M, Benett, N.C. and Schoeman, A.S. 1998. The social structure and dominance hierarchies of the Highveld mole-rat Cryptomys hottentotus pretoriae (Rodentia: Bathyergidae). Journal of Zoology, London. 246, 193-201

Oosthuizen, M.K., Bennett, N.C., Lutermann, H. and Coen, C.W. 2008. Reproductive suppression and the seasonality of reproduction in the social Natal mole-rat (Cryptomys hottentotus natalensis). General and Comparative Endocrinology. 159, 236-240.

Reeve, H.K. (1992). Queen activation of lazy workers in colonies of the eusocial naked mole-rat. Nature 358: 147-149.

Sichilima, A.M.., Bennett, N.C., Faulkes C.G.. \& Le Comber, S.C. (2008). Evolution of mammalian sociality: burrow architecture, rainfall and foraging in a social mole-rat. Journal of Zoology, London 275: 276-282.

Sichilima, A.M., Faulkes, C.G \& Bennett, N.C. (2008). Field evidence for aseasonality of reproduction and colony size in the Afrotropical giant mole-rat Fukomys mechowii (Rodentia: Bathyergidae). African Zoology 43: 144-149.

Snowden, C.T. 1996. Infant care in cooperatively breeding species. Advances in the Study of Behaviour. 25, 643-689

Vehrencamp, S.L. (1983). A model for the evolution of despotic versus egalitarian societies. Animal Behaviour 31: 667-682.

Wallace, E.D. and Bennett, N.C. 1998. The colony structure and social organization of the giant Zambian mole-rat, Cryptomys mechowi. Journal of Zoology, London. 244, 51-61.

Willingstorfer W.J., Burda, H., Winckler, J 1998. Ovarian growth and folliculogenesis in breeding and nonbreeding females of a social rodent, the Zambian common mole-rat, Cryptomys sp.. Journal of Morphology, 237, 33-41, 